\title{
Numerische Berechnung von Wirbelstromproblemen mit der Cell-Methode
}

\author{
K. Frenner and W. M. Rucker \\ Institut für Theorie der Elektrotechnik, Universität Stuttgart, Deutschland
}

Zusammenfassung. In dieser Arbeit wird die Cell-Methode auf die quasistatischen Maxwellgleichungen angewendet. Dabei werden für die notwendige Transformation vom Primärgitter auf das duale Gitter reziproke Basisvektoren verwendet. Anhand der Felddiffusion der magnetischen Induktion in einen zylindrischen Leiter werden Ergebnisse der Cell-Methode mit einer analytischen Vergleichsrechnung präsentiert.

\section{Einleitung}

Neben der Finiten-Integrations-Technik (Clemens, 2001) ist die Cell-Methode (Mattiussi, 2000) ein wichtiges Verfahren zur Berechnung elektromagnetischer Felder, das auf der integralen Formulierung der Maxwell-Gleichungen beruht.

Dabei werden statt den Feldgrößen, die an einem Punkt definiert sind, die sogenannten globalen Variablen betrachtet. Diese globalen Größen sind die Linien- oder Flächenintegrale der Feldgrößen auf dem Gitter.

Durch die globalen Größen zerfallen die Integrale der Maxwellgleichungen in Summen aus diesen globalen Variablen.

Mit den globalen Größen

- Fluss der magnetischen Induktion $B$ durch eine Fläche $B_{s}=\int_{s} \mathbf{B} \cdot d s$

- Linienintegral des elektrischen Feldes $E$ entlang einer Kante $E_{k}=\int_{k} \mathbf{E} \cdot d k$

- Fluss der Stromdichte $J$ durch eine Fläche $J_{\bar{s}}=\int_{\bar{s}} \mathbf{J} \cdot d \bar{s}$

- Linienintegral des Magnetfeldes $H$ entlang einer Kante $H_{\bar{k}}=\int_{\bar{k}} H \cdot d \bar{k}$

Correspondence to: K. Frenner

(karsten.frenner@ite.uni-stuttgart.de)

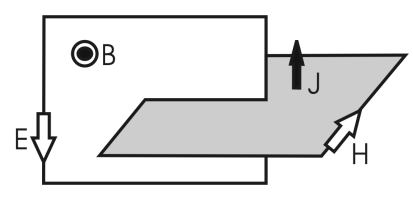

Abbildung 1. Struktur der Maxwellgleichungen im quasistatischen Fall.

lauten die dazugehörigen Maxwellgleichungen:

$\begin{aligned} \sum_{\bar{k}} H_{\bar{k}} & =J_{\bar{s}} \\ \sum_{k} E_{k} & =-\partial_{t} B_{s}\end{aligned}$

Diese zwei Gleichungen lassen sich also mit Hilfe der globalen Größen exakt darstellen. Nehmen wir die Materialgleichungen

$\mathbf{J}=\kappa \mathbf{E}$

$\mathbf{B}=\mu \mathbf{H}$

erhalten wir miteinander verkettete Linienintegrale. (Abb. 1)

Um diese verkettete Struktur nachzubilden, müssen wir die auftretenden Größen auf zwei ineinander verschachtelte Gitter verteilen. Das führt auf ein Primärgitter mit den Kanten $k$ und den Seiten $s$ und dem dualen Gitter mit den dualen Kanten $\bar{k}$ und Seiten $\bar{s}$.

Im zweidimensionalen Fall (Abb. 2) sieht man, dass jeweils ein Knoten des primären Gitters von dualen Kanten umgeben ist und umgekehrt.

Es gibt verschiedene Möglichkeiten ein duales Gitter zu konstruieren. In dieser Arbeit wird das baryzentrische Gitter verwendet: Die dualen Knoten der Elemente befinden sich im Schwerpunkt der primären Elemente, die dualen Kanten gehen vom dualen Knoten zu den Mittelpunkten der primären Kanten und spannen eine (unregelmäßig geformte) duale

Published by Copernicus GmbH on behalf of the URSI Landesausschuss in der Bundesrepublik Deutschland e.V. 


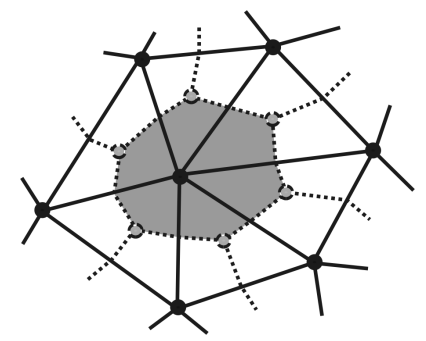

Abbildung 2. Gitterausschnitt mit primären Dreieckselementen und einem dualen Element.

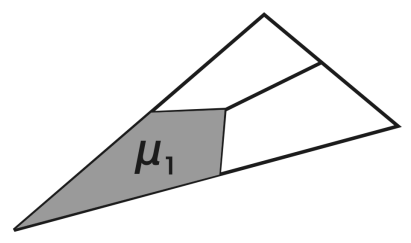

Abbildung 3. Unterteilung eines Dreiecks in drei Mikrozellen. Hervorgehoben ist die Mikrozelle $\mu_{1}$.

Fläche auf. Eine Diskussion anderer dualer Gitter findet sich z.B. in Tonti (2002).

Durch die unregelmäßige Form der dualen Elemente ist es von Vorteil, wenn man die primären Elemente in Mikrozellen unterteilt. Eine Mikrozelle ist dabei genau in einem primären und einem dualen Element enthalten. Damit lässt sich beim Aufstellen der Systemgleichungen eine Summe über alle primären Elemente bilden. Die Mikrozelle ist der Anteil eines dualen Elementes, der ein primäres Element enthält. Alle Mikrozellen haben bei zweidimensionalen Gittern die Form von Vierecken (Abb. 3) oder Hexaedern im dreidimensionalen Fall (Abb. 4).

\section{Aufstellen der Systemgleichungen}

Für das numerische Beipiel im Abschnitt 3 benötigen wir ein zweidimensonales Modell, da die analytische Vergleichsrechnung nur für einen unendlich ausgedehnten Zylinder zur Verfügung steht. Der Zylinder sei hier in z-Richtung unendlich ausgedehnt. Wir beschränken uns im Folgenden auf die Herleitung der Systemgleichungen für diesen Fall, in der Arbeit Brinner (2005) sind Beispiele für dreidimensionale Modelle berechnet.

Wir gehen von einem homogenen Material mit der Permeabilität $\mu$ und dem spezifischen ohmschen Leitwert $\kappa$ aus und betrachten ein beliebiges Element (Abb. 5). Es besitzt drei primäre Kanten $k_{1}, k_{2}$ und $k_{3}$. Die Integrale der elektrischen Feldstärken $E_{p_{1}}, E_{p_{2}}$ und $E_{p_{3}}$ ordnen wir aber nicht den Kanten, sondern den primären Knoten $p_{1}, p_{2}$ und $p_{3}$ $\mathrm{zu}$, denn das elektrische Feld hat in diesem Fall nur eine z-Komponente. Senkrecht auf den primären Kanten stehen

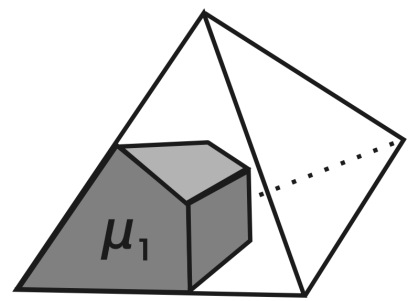

Abbildung 4. Unterteilung eines Tetraeders in vier Mikrozellen. Dargestellt ist die Mikrozelle $\mu_{1}$.

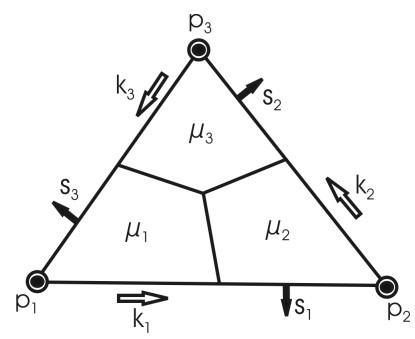

Abbildung 5. Dreieckselement mit primären Kanten und Seiten.

die Vektoren $s_{1}, s_{2}$ und $s_{3}$, mit denen wir die magnetischen Flüsse $B_{s_{1}}, B_{s_{2}}$ und $B_{s_{3}}$ darstellen.

Für die dualen Größen betrachten wir die Mikrozelle $\mu_{1}$ eines beliebigen Elementes (Abb. 6). Dort werden den dualen Kanten $\bar{k}_{1}$ und $\bar{k}_{2}$ die Linienintegrale der magnetischen Feldstärke $H_{\bar{k}_{1}}$ und $H_{\bar{k}_{2}}$ zugeordnet. Dem Strom $J_{\bar{s}_{\mu}}$ entspricht der Anteil der dualen Fläche von $\mu_{1}$ an der zum Knoten $p_{1}$ zugeordneten dualen Fläche.

Wir betrachten jetzt den Fluss $B_{s_{1}}$. Für die Zeitableitung gilt gemäß Gl. (2):

$-\partial_{t} B_{s_{1}}=-E_{p_{1}}+E_{p_{2}}$

Wir berücksichtigen vorerst nur den Integrationsweg entlang der dualen Kanten von $\mu_{1}$. Der Anteil $E_{p_{2}}$, der am Rand von $\mu_{2}$ entlangführt, muss dann beim Assemblieren der Gesamtmatrix noch addiert werden. Mit dem Materialgesetz (3) und dem Knoten $p_{1}$ zugeordneten dualen Flächeninhalt $A_{p_{1}}$ erhalten wir

$-\partial_{t} B_{\mu_{1}, s_{1}}=-\frac{1}{\kappa A_{p_{1}}} J_{p_{1}}$.

Mit Gl. (1) folgt daraus

$-\partial_{t} B_{\mu_{1}, s_{1}}=-\frac{1}{\kappa A_{p_{1}}} \sum_{\bar{k}} H_{\bar{k}}$.

Jetzt müssen die Feldstärken $H_{\bar{k}}$ durch die Flüsse $B_{\mu_{1}, s_{i}}$ ausgedrückt werden. Dafür benutzen wir Gl. (4) und rekonstruieren den Vektor $\mathbf{B}$ aus den Projektionen $B_{\mu_{1}, s_{i}}$ mit Hilfe reziproker Gittervektoren $s^{i}$ (Abb. 7). Es gibt auch andere Möglichkeiten zur Transformation auf das duale Gitter, z.B. 


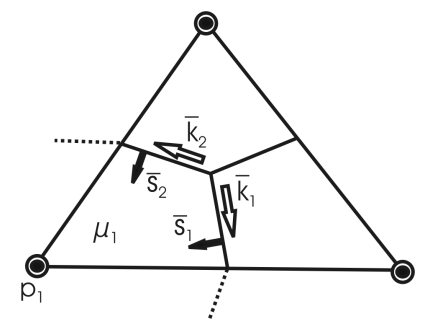

Abbildung 6. Element mit dualen Kanten und Seiten.

kann man die Projektionen $B_{\mu_{1}, s_{i}}$ mit Hilfe von Formfunktionen interpolieren (Repetto, 2003) oder die Rekonstruktion durch eine einfache Mittelwertbildung erreichen (Brinner, 2005).

Die reziproken Gittervektoren in Abb. 7 sind gegeben durch (Ibach, 2000)

$s^{1}=\frac{s_{3} \times e_{z}}{s_{1} \cdot\left(s_{3} \times e_{z}\right)}$

$s^{3}=-\frac{s_{1} \times e_{z}}{s_{1} \cdot\left(s_{3} \times e_{z}\right)}$

Damit bekommen wir:

$$
-\partial_{t} B_{\mu_{1}, s_{1}}=-\frac{1}{\kappa A_{p_{1}}} \sum_{\bar{k}_{i}}\left(B_{s_{1}} s^{1}+B_{s_{3}} s^{3}\right) \cdot \bar{k}_{i}
$$

Das ist der Anteil der Mikrozelle $\mu_{1}$ an der Zeitableitung. Ebenso verfährt man mit allen an $p_{1}$ angrenzenden Mikrozellen und summiert dann alle Anteile.

Für die Zeitableitung des Flusses $B_{s_{1}}$ müssen dann die Anteile beider Knoten $P_{1}$ und $P_{2}$ berücksichtigt werden:

$$
\begin{aligned}
-\partial_{t} B_{s_{1}} & =-\frac{1}{\kappa A_{p_{1}}} \sum_{\mu_{i}} \sum_{\bar{k}_{i}}\left(B_{s_{1}} s^{1}+B_{s_{3}} s^{3}\right) \cdot \bar{k}_{i} \\
& +\frac{1}{\kappa A_{p_{2}}} \sum_{\mu_{j}} \sum_{\overline{k_{j}}}\left(B_{s_{1}} s^{1}+B_{s_{2}} s^{2}\right) \cdot \bar{k}_{j}
\end{aligned}
$$

Für alle Flüsse $B_{i}$, die nicht durch die Randbedingungen auf einen festen Wert gesetzt sind, kann man so eine Gleichung aufstellen. Alle mit den Randknoten verbundenen Kanten werden auf einen vorgegebenen Randwert gesetzt, diese Werte werden in die Gleichungen eingesetzt und die dabei entstehenden Koeffizienten auf die rechte Seite gebracht. Man erhält ein Gleichungssystem der Form:

$\partial_{t} B_{i}+\sum_{j} S_{i j} B_{j}=f_{i}$

Für die Zeitintegration stehen verschiedene Algorithmen zur Verfügung (Mattiussi, 2001). Das folgende numerische Beispiel wurde mit dem impliziten Trapezverfahren mit der Schrittweite $\Delta t=0.023$ erstellt.

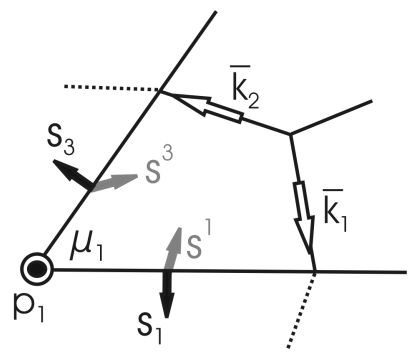

Abbildung 7. Für die Materialgesetze: Rekonstruktion eines Vektors aus den Flächenintegralen der Seitenflächen und anschliessende Projektion auf die dualen Kanten mit Hilfe der reziproken Basisvektoren $s^{1}$ und $s^{2}$.

Es folgt dann für die Zeitentwicklung:

$\sum_{j}\left(\delta_{i j}+\frac{\Delta t}{2} S_{i j}\right) B_{j}^{n+1}=\sum_{j}\left(\delta_{i j}-\frac{\Delta t}{2} S_{i j}\right) B_{i}^{n}+f_{i}$

In jedem Zeitschritt $n$ muss also ein Gleichungssystem gelöst werden.

Für das Randwertproblem aus dem Abschnitt 3 werden als Anfangsbedingung für den Zeitschritt $B_{i}^{0}$ die Werte im Inneren des Zylinders auf Null gesetzt.

\section{Numerisches Beispiel}

Gegeben ist ein in z-Richtung unendlich ausgedehnter Metallzylinder mit Radius $r=1$. Das Modell wurde mit ca. 3000 linearen Dreieckselementen vernetzt. Als Randbedingung wurde ein zeitlich konstantes azimutales Feld vom Betrag $B_{\phi}=1$ angelegt. Das Innere des Zylinders ist zunächst feldfrei.

Das B-Feld beginnt in den Zylinder zu diffundieren (Abb. 8-11). Dieser Vorgang läßt sich durch eine Reihenentwicklung analytisch beschreiben (Lehner, 1990). Diese Reihenentwicklung der Lösung wurde mit bis zu 300 Summanden ausgewertet und zum Vergleich der Cell-Rechnungen verwendet.

Bei der Cell-Rechnung sind die Feldwerte in den Elementen konstant. Die auftretenden Abweichungen zur Vergleichslösung sind durch die Gitterweite bedingt.

Für die Rechnungen wurden dimensionslose Größen für die Zeit $t$ und den Radius $r$ verwendet. Durch die Transformation

$$
\begin{aligned}
& x=\frac{\bar{x}}{r} \\
& t=\frac{\bar{t}}{\mu \kappa r^{2}}
\end{aligned}
$$

läßt sich die Lösung auf beliebige Radien transformieren. 


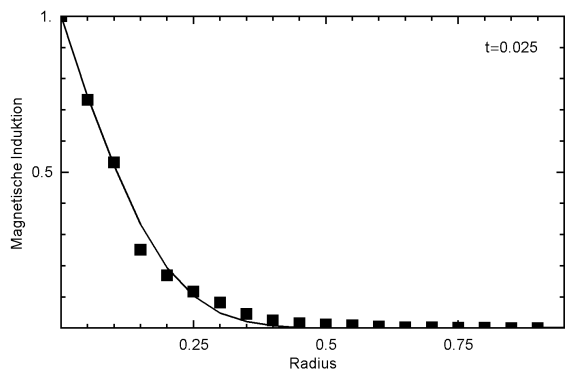

Abbildung 8. Diffusion der magnetischen Induktion in einen unendlich langen zylindrischen Leiter zum Zeitpunkt $t=0.025$. Die Randbedingung ist ein azimutales Feld vom Betrag 1. Eingezeichnet ist die Lösung der Cell-Methode (Quadrate) und eine analytische Vergleichsrechnung (durchgezogene Linie). Die Zeit, der Radius und die magnetische Induktion sind hier dimensionslose Größen.

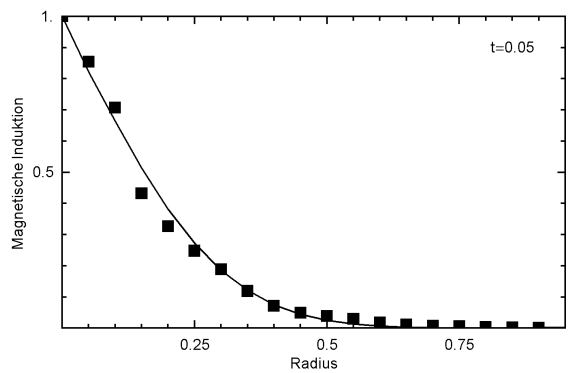

Abbildung 9. Betrag der magnetischen Induktion zum Zeitpunkt $t=0.05$.

\section{Zusammenfassung}

In dieser Arbeit wurde die Cell-Methode auf ein Wirbelstromproblem, nämlich der Felddiffusion in einen unendlich ausgedehnten Zylinder, angewendet. Auf dem gegebenen Primärgitter aus linearen Dreieckselementen wurde ein baryzentrisches duales Gitter entworfen. Es werden globale Variablen eingeführt, mit denen ein Teil der physikalischen Gesetze exakt in diskreter Form wiedergegeben werden kann. Für die Materialgleichungen wird mit Hilfe reziproker Gittervektoren eine Transformation der primären Größen auf das duale Gitter hergeleitet. Die berechneten Felder stimmen gut mit einer analytischen Vergleichslösung überein.

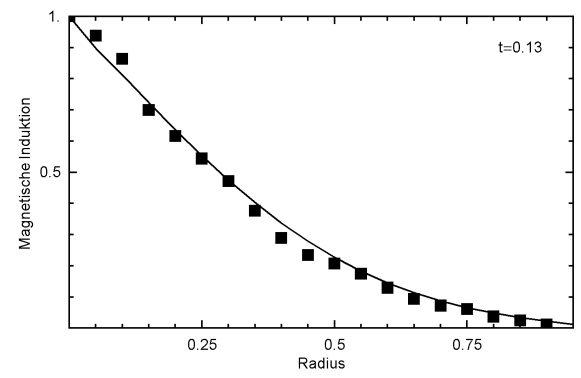

Abbildung 10. Betrag der magnetischen Induktion zum Zeitpunkt $t=0.13$.

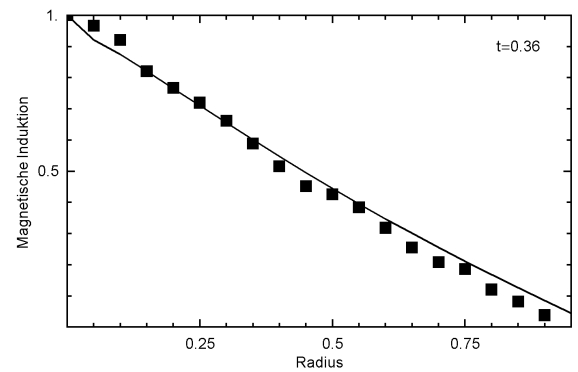

Abbildung 11. Betrag der magnetischen Induktion zum Zeitpunkt $t=0.36$

\section{Literatur}

Brinner, A.: Numerische Berechnung von Wirbelstromproblemen mit einem Clifford-Cell Algorithmus, Diplomarbeit, Universität Stuttgart 2005.

Clemens, M. and Weiland, T.: Discrete electromagnetism with the finite integration technique, PIER monograph series, 32, 65-87, 2001.

Ibach, H. and Lüth, H.: Festkörperphysik: Einführung in die Grundlagen, Springer, Berlin Heidelberg, 44-46, 1995.

Lehner, G.: Elektromagnetische Feldtheorie für Ingenieure und Physiker, Springer, Berlin Heidelberg, 328 ff, 1990.

Mattiussi, C.: The Finite Volume, Finite Difference, and Finite Elements Methods as Numerical Methods for Physical Field Problems, Adv. in Imaging and Elektron Phys., edited by Hawkes, P., 113, 1-146, 2000.

Mattiussi, C.: The Geometry of Time-Stepping, Progress in Electromagn. Res., PIER, 32, 123-149, 2001.

Repetto, M. and Trevisan, F.: 3-D Magnetostatic With the Finite Formulation, IEEE Transactions on Magnetics, 39, 1135-1138, 2003.

Tonti, E.: Finite Formulation of Electromagnetic Field, IEEE Transactions on Magnetics, 38, 333-336, 2002. 